\title{
HRQoL in Barth Syndrome: Agreement between Child Self-reports and Parent Proxy-reports and Its Relationship to Parental HRQoL
}

\author{
Yoonjeong Lim ${ }^{1 *}$, Consuelo M. Kreider ${ }^{2}$, Mary Alvarez', Roxanna M. Bendixen ${ }^{3}$
}

'Department of Occupational Therapy Byrdine F. Lewis College of Nursing \& Health Professions, Georgia State University, Atlanta, GA, USA

${ }^{2}$ Department of Occupational Therapy, College of Public Health and Health Professions, University of Florida, Gainesville, FL, USA

${ }^{3}$ Department of Occupational Therapy, School of Health and Rehabilitation Sciences, University of Pittsburgh, Pittsburgh, PA, USA

Article Info

\section{Article Notes}

Received: March 31, 2019

Accepted: June 13, 2019

\section{*Correspondence:}

Dr. Yoonjeong Lim, Department of Occupational Therapy Byrdine F. Lewis College of Nursing \& Health Professions, Georgia State University, Atlanta, GA, USA;

Email: ylim@gsu.edu.

${ }^{\circledR} 2019 \operatorname{Lim}$ Y. This article is distributed under the terms of the Creative Commons Attribution 4.0 International License.

\section{Keywords:}

Barth syndrome

Health-related quality of life

HRQoL

Proxy-report

Rare Diseases

Self-report

\section{Abstract}

Purpose: Barth syndrome is an X-linked rare disorder that typically affects only males. This study investigates 1 ) agreement between child self-reports and parent proxy-reports of HRQoL in boys with Barth syndrome and 2) relationship between parental HRQoL and parent proxy-reports of HRQoL for the child.

Materials and methods: Twenty-eight boys with Barth syndrome and their parents participated in this study. The PedsQL ${ }^{\mathrm{TM}} 4.0$ and the PedsQL ${ }^{\mathrm{TM}}$ Family Impact Module were used to measure HRQoL of the boys, and the parents' HRQoL, respectively. The Intraclass Correlation Coefficient was used to test agreement between the child self-reports and parent proxy-reports of HRQoL. The Spearman correlation coefficient was used to test the relationship between parental HRQoL and parent proxy-reports of HRQoL for the child.

Results: The agreement between the child self-reports and the parent proxy-reports showed moderate-to-good agreement. Higher parental HRQoL was significantly related to higher ratings of the parents on their children's HRQoL $(p<.05)$.

Conclusions: This study broadens understanding of HRQoL of boys with Barth syndrome using both child self-reports and parent proxy-reports. The findings indicate that the parent proxy-report of HRQoL should be used in conjunction with the child self-report when making client-centered health decisions.

\section{Introduction}

Barth syndrome (BTHS) is a rare, life-threatening X-linked genetic disorder that typically affects only males ${ }^{1}$. First described in 1983, BTHS has been diagnosed in fewer than 500 living males worldwide; however, evidence suggests that BTHS may be significantly underdiagnosed due to the wide variety of clinical features associated with the disorder ${ }^{2}$. Therefore, the exact prevalence of BTHS remains unknown, though the disorder is estimated to occur in roughly 1 out of every 300,000 - 400,000 U.S. births ${ }^{2,3}$. Once regarded as primarily a cardiac condition, BTHS is now recognized as a multi-system disorder caused by a complex inborn metabolic error ${ }^{1,2,4}$.

While the exact nature and degree of symptoms vary considerably in different individuals, cardinal characteristics of BTHS include cardiomyopathy, neutropenia, muscle hypoplasia and weakness, growth delays and short stature, exercise intolerance, 3-methylglutaconic aciduria, and cardiolipin deficiency ${ }^{5,6}$. These symptoms are typically detected in infancy or early childhood, though diagnosis of BTHS is often difficult or delayed ${ }^{3,7}$. Though 
survival rates have improved significantly in recent years, individuals with BTHS remain predisposed to premature death due to cardiac issues and risk of infection related to neutropenia ${ }^{5,8}$. BTHS is frequently associated with physical effects, such as fatigue, pain, and feeding problems, as well as mild cognitive effects on visuo-spatial skills and motor learning $7,9,10,11,12$. These cognitive and physical effects may contribute to increased functional difficulties in academics, prevocational/vocational activities, and independent living skills ${ }^{13}$. In addition, boys with BTHS have been reported to have difficulty getting along with peers, often experiencing bullying because of their small stature and limits on physical exertion ${ }^{11,14}$. These peer interactions contribute to increased stress and may lead to lowered self-esteem ${ }^{14}$.

Given the impact of BTHS on an individual's functioning and well-being, it is understandable that initial research indicates that boys with BTHS have significantly reduced quality of life as related to their health ${ }^{11}$. Health-related quality of life (HRQoL) is a multidimensional construct that considers the impact of disease or health conditions on overall health and daily living ${ }^{15,16}$. Physical, emotional, and social functioning are considered when assessing HRQoL ${ }^{16}$. When a child can self-report his or her health status, child self-report is typically used ${ }^{17}$. However, if the child is very young or cannot complete the self-report due to illness or cognitive impairment, parents may be asked to report on their child's HRQoL via parent proxy-report ${ }^{18,19}$. While some earlier studies misguidedly attempted to determine whose report was more "accurate", current research typically accepts that both the parent and child reports provide invaluable information and that comprehensive evaluation should include consideration of both the child and parent perspectives ${ }^{17}$.

Previous studies of children with chronic conditions report varying degrees of agreement between child selfreports and parent proxy-reports of HRQoL ${ }^{20,21,22}$. However, in general, parents of children with chronic conditions typically report lower HRQoL for the children than the children themselves ${ }^{23,24,25,26}$. This contrasts with parents of healthy children, who tend to rate their children's HRQoL higher than the children themselves ${ }^{15,17}$. By identifying which aspects of HRQoL show the greatest discrepancies in the level of agreement between child self-reports and parent proxy-reports of HRQoL, clinicians can better understand the differences in perspectives of HRQoL between a child and his or her parents ${ }^{20,27}$. This understanding may then provide useful information for guiding clinical decision making, such as when planning rehabilitation therapy goals.

Research suggests that the parent's own psychosocial health may be particularly impactful on their perception of their child's quality of life ${ }^{25,28}$. Indeed, parents with higher perceived levels of parenting burden ${ }^{20}$ and distress ${ }^{29}$ generally provide lower ratings for their child's HRQoL. Thus, the parent's own HRQoL, including psychosocial factors, should also be considered when using parentproxy reports or when investigating discrepancies between parent-proxy and child self-reports. This is particularly important for parents of children with BTHS, who may experience increased stress from dealing with the medical management of the condition, as well as emotional impact due to concerns about the child's future and mortality ${ }^{13}$. Furthermore, assessing a parent's HRQoL can help identify ways to better support the parent in caring for their child.

There have been very few studies focusing on the HRQoL of children with BTHS, though the psychosocial functioning of children with BTHS and their parents has been studied by

Storch and colleagues ${ }^{13}$ and Jacob and colleagues ${ }^{11}$. However, no prior studies have specifically focused on the agreement between child self-reports and parent proxyreports of HRQoL in boys with BTHS. This is particularly important because research suggests that the nature and extent of discrepancies in parent-proxy reports and child self-reports of HRQoL vary depending on the condition ${ }^{17}$. Consequently, these factors must be investigated specifically with BTHS in order to best serve the needs of children with BTHS and their families. Therefore, the purpose of this study is to: 1) examine the agreement between child selfreports and parent proxy-reports of HRQoL for boys with BTHS and 2) investigate the relationship between parental HRQoL and parent proxy-reports of HRQoL for the child. We anticipate that parents' own HRQoL will have positive relationships to the boys' HRQoL as rated by the parents.

\section{Methods}

A two-group cross-sectional design was used to investigate 1) agreement between child self-reports and parent proxy-reports of HRQoL in boys with Barth syndrome (BTHS); and 2) relationship between parental HRQoL and parent proxy-reports of HRQoL for the child. Participants were boys with BTHS, ages $4-17$ (10.70 \pm $3.70 ; \mathrm{N}=28$ ) and one parent of each child ( $\mathrm{N}=28)$. Five children fell in the age group of 4 to 7,12 children in the age 8-to-12 group, and 11 children in the age 13-to-17 group. Participants were recruited at the 2010 and/or 2012 Barth Syndrome International Conference. This conference is a scientific, medical, and family conference held in the United States every two years. The conference was attended by families from the US, Canada, United Kingdom, and France. For participants who attended both the 2010 and 2012 Conferences, only data from the 2012 Conference were used.

This study was approved by the University of Florida's Institutional Review Board. Before data collection, written informed consent was obtained from the parent, and assent obtained from the boy. Boys with BTHS completed 
an HRQoL self-report, and one of the parents completed the same HRQoL instrument using the parent proxy-report version.

\section{Measures}

Pediatric quality of life inventory version 4.0: The Pediatric Quality of Life Inventory Version 4.0 (PedsQL ${ }^{\mathrm{TM}}$ ) was used to measure the HRQoL of boys with BTHS. Both the child report and the parent report versions were used in this study. The PedsQL ${ }^{\mathrm{TM}}$ consists of four subscales: Physical Functioning (8 items), Emotional Functioning (5 items), Social Functioning (5 items), and School Functioning ( 5 items) ${ }^{30}$. Subscale scores are calculated by scoring each item on a 5 -point scale (never $=100$; almost never $=75$; sometimes $=50$; often $=25$; almost always $=$ $0)$, then averaged within each subscale. The total score is the mean of all items across the four subscales with higher scores indicating better HRQoL. The reliability and validity of the PedsQL ${ }^{\mathrm{TM}}$ were established using 963 children with or without chronic illness and 1,629 parents ${ }^{31}$. Clinical cut points are established for the total score between 71 and 76 for children with moderate chronic conditions, and 70 or below for children with major chronic conditions ${ }^{32}$. The PedsQL ${ }^{\mathrm{TM}}$ subscale scores (e.g., physical functioning) and total score were used as variables in the analyses.

PedsQL $L^{\mathrm{TM}}$ family impact module: The PedsQL $\mathrm{L}^{\mathrm{TM}}$ Family Impact Module (PedsQL ${ }^{\mathrm{TM}} \mathrm{FI}$ ) was used to assess parental quality of life (QoL). The PedsQL ${ }^{\mathrm{TM}}$ FI is a 36item questionnaire measuring the domains of Parental QoL and Family Functioning ${ }^{33}$. Parental QoL was used as a variable and calculated as the mean of items from the subscales: 1) Physical Functioning (6 items), 2) Emotional Functioning (5 items), 3) Social Functioning (4 items), and 4) Cognitive Functioning (5 items). In this instrument, each item is scored on a 5 -point scale (never a problem = 100; almost never $=75$; sometimes $=50$; often $=25$; almost always $=0$ ) with higher scores representing better parental QoL. Evidence of reliability and validity of the PedsQL ${ }^{\mathrm{TM}}$ FI instrument was established with 458 parents of children or adolescents with chronic pain ${ }^{34}$. Statistical analysis
Distributions of the data were tested using the ShapiroWilk Test. Levels of agreement between child and parent reports were tested using a Two-Way Mixed-Effect Model (absolute agreement, average measures) Intraclass Correlation Coefficient (ICC). ICC value of $\leq 0.4$ indicates poor-to-fair agreement, 0.41 to 0.60 means moderate agreement, 0.61 to 0.80 is good agreement, and 0.81 to 1.00 represents excellent agreement ${ }^{35}$. Magnitude of mean differences in child self-reports and parent proxy-reports were analyzed using paired $t$-tests. Consistency in rank order relationships between the child and parent reports were tested using the Pearson Correlation Coefficient (Pearson $r$ ). Spearman Correlation Coefficient $\left(r_{s}\right)$ was used to test the relationship between parental HRQoL and parent-rated HRQoL of the child. SPSS version 24 was used for all data analyses ( $\alpha=0.05 ; 2$-tailed assumed).

\section{Results}

All data were normally distributed as tested using the Shapiro-Wilk Test ( $p>.05)$. Findings from the ICC indicate that the agreement between child self-reports and parent proxy-reports was moderate-to-good. The results of the agreement in the ICC were good between the child selfreports and parent proxy-reports of HRQoL for Physical Functioning, Emotional Functioning, School Functioning, and Total Score. Moderate agreement was found in Social Functioning (Table 1).

The means of self-reports of children with BTHS were significantly higher than those of parent proxy-reports for all the scales and Total Score $(p<.05)$. Magnitude of PedsQL ${ }^{\mathrm{TM}}$ mean scores were significantly different between child self-reports and parent proxy-reports in Physical Functioning $(t(27)=3.267, p=.003)$, Emotional Functioning $(t(27)=2.319, p=.028)$, Social Functioning $(t(27)=3.377, p=.002)$, and School Functioning $(t(27)=$ 2.559, $p=.016)$, as well as Total Score $(t(27)=4.386, p<$ .001). Parent proxy scores were lower than child reported scores across all subscales and Total Score (Table 1). With regard to rank order of the ratings, the Pearson $r$ was significant for the four subscales and total score. The child self-reports and parent proxy-reports were consistent

Table 1. HRQoL mean scores and the agreement between children and parents.

\begin{tabular}{|c|c|c|c|c|c|c|}
\hline & Child self-report & Parent proxy-report & \multirow{2}{*}{$p$} & \multirow{2}{*}{$\mathrm{Cl}^{++}$} & \multirow{2}{*}{ Pearson $r$} & \multirow{2}{*}{$\mathrm{ICC}^{+}$} \\
\hline & Mean (SD) & Mean (SD) & & & & \\
\hline Physical Functioning* & $62.28(21.98)$ & $49.89(24.26)$ & .003 & {$[4.60,20.17]$} & $.627^{* *}$ & .712 \\
\hline Emotional Functioning* & $70.18(17.13)$ & $63.21(18.27)$ & .028 & {$[.80,13.13]$} & $.599^{* *}$ & .720 \\
\hline Social Functioning* & $70.89(17.90)$ & $58.11(18.66)$ & .002 & {$[5.02,20.55]$} & $.400^{*}$ & .493 \\
\hline School Functioning ${ }^{*}$ & $66.61(21.90)$ & $55.71(23.56)$ & .016 & {$[2.16,19.63]$} & $.511^{* *}$ & .634 \\
\hline Total Score ${ }^{*}$ & $66.81(17.31)$ & $55.82(15.96)$ & $<.001$ & {$[5.85,16.13]$} & $.685^{* *}$ & .723 \\
\hline
\end{tabular}

${ }^{*} p<0.05$. ${ }^{* *} p<0.01$.

+ Intraclass correlation coefficient of $\leq 0.40=$ poor-to-fair agreement, 0.41 to $0.60=$ moderate agreement, 0.61 to $0.80=$ good agreement, and 0.81 to $1.00=$ excellent agreement ${ }^{35}$.

${ }^{++} 95 \%$ confidence interval of the difference 
despite differences in the magnitude of responses (i.e., ratings) (Table 1).

A positive relationship was found between parental HRQoL and parent proxy-reports of HRQoL for the child $\left(r_{s}(26)=.581, p=.001\right)$. Thus, the higher the scores for parental HRQoL, the higher the scores for parent proxyreports of HRQoL for the child.

\section{Discussion}

This study investigated the extent of agreement in perceptions of HRQoL reported by boys with BTHS compared to the parent's perception of the boy's HRQoL. The agreement found in our study was higher than those previously reported in the literature ${ }^{35,36,37,38}$. In our study, we found good agreement for physical functioning, emotional functioning, and school functioning in boys with BTHS, whereas there was only moderate agreement for social functioning. Consistent with the literature, we observed higher agreement for physical functioning than for social functioning $39,40,41$. These findings suggest that parents may be more knowledgeable of their child's physical status than of the child's social experiences.

Children with disabilities often have constrained social participation ${ }^{42}$. Boys with BTHS have difficulty participating in activities requiring physical exertion, which can hinder socialization ${ }^{14}$. Bullying experiences related to small stature and chronic pain ${ }^{13}$ and potential learning difficulties and sensory issues in BTHS can also impact socially-related quality of life. Lower agreements in ratings of social functioning suggest that parents may not fully appreciate the intricacies of their child's social experiences. Good understanding of the child's social experiences is important for decision making when considering ways to support or facilitate the child's social engagement. The lower agreements in ratings of social functioning (as compared to physical functioning) point to the importance of incorporating measurement of both child self-reports and parent proxy-reports of HRQoL into research and clinical practice.

In this study, parents perceived the boys' HRQoL to be lower than that reported by the boys themselves. This is consistent with previous research in children with chronic diseases, which found that parents tend to underestimate their child's HRQoL ${ }^{35,43}$. Findings may reflect parental concern regarding perceived impacts of physical difficulties on their child's daily life and overall HRQoL. However, findings might also suggest the boys' reduced awareness of the impacts related to their impaired health status ${ }^{35}$, or the presence of beneficial coping and/or compensatory strategies. Boys with BTHS grow up with physical symptoms that manifest at young ages ${ }^{4}$, thus creating opportunity for adjustment to physical constraints early in development. Nonetheless, the mean scores as reported by both the boys and their parents fell below clinically meaningful cutoff scores compared to moderate and major chronic health conditions (76 and 70 respectively) ${ }^{32}$. Therefore, our findings contribute evidence of diminished HRQoL for children growing up with BTHS.

In addition, we found a significant positive relationship between parental HRQoL and parent proxy-reports of HRQoL for the child, indicating higher parental HRQoL was significantly related to higher ratings of the parents on their children's HRQoL. The finding is consistent with other studies that reported associations between parent proxy-report of HRQoL for the child and parental HRQoL ${ }^{28}$, parental stress $^{44}$, and parental health ${ }^{45}$. Our finding contributes to the existing evidence that points to the importance of assessing the parent's own HRQoL when the parent rates the child's HRQoL.

Client-centered care concentrates on the child's needs and functioning rather than focusing solely on the disease; such care contributes to high-quality, desirable, and effective healthcare services ${ }^{46}$. Assessing the child's HRQoL provides valuable understanding for client-centered care. Quality of life is inherently subjective, and the child's perceptions provide salient information. Therefore, children who can report on their perceived quality of life should report themselves. However, parent proxy-reports of HRQoL can provide information different from that reported by the child. Therefore, parent proxy-reports should be used in conjunction with the child self-report when making clientcentered health decisions. This is because parent proxyreports remain an important source of complementary information about the child's HRQoL.

\section{Conclusion}

This study broadens understanding of HRQoL of boys with BTHS using both child self-reports and parent proxyreports. HRQoL measurement using both child self-reports and parent proxy-reports can contribute to demonstrating the value of interventions in research and clinical practice. Our sample is not large enough to generalize results. However, it is notable that according to the BTHS registry ${ }^{4}$, our sample size of 28 children represents almost $6 \%$ of the known population of individuals with BTHS. Sample size hindered our ability to assess potential impacts of parenting role (e.g., mother versus father) on proxy-reporting. Future research should include investigation of factors such as the child's age, disease severity, family functioning, and family cohesion in order to elucidate child and parental factors affecting level of agreement between child self-reports and parent proxy-reports.

\section{Acknowledgments}

The project described here was supported in part by Award Number K01HD064778 and K12HD055929 from the Eunice Kennedy Shriver National Institute of Child 
Health and Human Development and the National Institute of Neurological Disorders and Stroke. The content is solely the responsibility of the authors and does not necessarily represent the official views of the National Institutes of Health. We appreciate the Barth Syndrome Foundation for their help in recruiting participants for this study. We are also deeply thankful to the participating parents and children for their unwavering support and devotion.

\section{References}

1. Barth Syndrome Foundation. Healthcare professional brochure: Do you know about Barth syndrome? [Internet]. Larchmonth (NY): Barth Syndrome Foundation; May 2016 [23 November 2017]. Available from: https://www.barthsyndrome.org/file_download/6c6439bc6d37-475b-97a5-68e39aa7907a.

2. Clarke SL, Bowron A, Gonzalez IL, et al. Barth syndrome. Orphanet J Rare Dis. 2013; 8(23). http://doi.org/10.1186/1750-1172-8-23

3. Roberts AE, Nixon C, Steward CG, et al. The Barth Syndrome Registry: Distinguishing disease characteristics and growth data from a longitudinal study. Am J Med Genet A. 2012; 158(11): 2726-2732.

4. Jefferies JL. Barth syndrome. Am J Med Genet C. 2013; 163(3): 198205.

5. Barth Syndrome Foundation. What is Barth syndrome [Internet]. Larchmonth (NY): Barth Syndrome Foundation; 2017 [11 December 2017]. Available from: https://www.barthsyndrome.org/ barthsyndrome/.

6. Bowron A, Honeychurch J, Williams $\mathrm{M}$, et al. Barth syndrome without tetralinoleoyl cardiolipin deficiency: A possible ameliorated phenotype. J Inherit Metab Dis. 2015; 38(2): 279-286.

7. Mazzocco MM, Henry AE, Kelly RI. Barth syndrome is associated with a cognitive phenotype. J Dev Behav Pediatr. 2007; 28(1): 22-30. doi:10.1097/01.DBP.0000257519.79803.90

8. Rigaud C, Lebre AS, Touraine R, et al. Natural history of Barth syndrome: A national cohort study of 22 patients. Orphanet J Rare Dis. 2013; 8(70). http://doi.org/10.1186/1750-1172-8-70

9. Reynolds S. Successful management of Barth syndrome: A systematic review highlighting the importance of a flexible and multidisciplinary approach. J Multidiscip Healthc. 2015; 8: 345-358. http://doi. org/10.2147/JMDH.S54802

10. Taylor D, Brady JE, Li G, et al. Characterization of pain in patients with Barth syndrome. J Child Health Care. 2016; 45(2): 192-203. https:// doi.org/10.1080/02739615.2014.996882

11. Jacob ML, Johnco C, Dane BF, et al. Psychosocial functioning in Barth syndrome: Assessment of individual and parental adjustment. J Child Health Care. 2017; 46(1): 66-92.https://doi.org/10.1080/02739615 .2015 .1124768

12. Raches D, Mazzocco MM. Emergence and nature of mathematical difficulties in young children with Barth syndrome. J Dev Behav Pediatr. 2012; 33(4): 328-335.

13. Storch EA, Keeley M, Merlo LJ, et al. (2009). Psychosocial functioning in youth with Barth syndrome. J Child Health Care. 2009; 38(2): 137156.

14. Keeley M, Merlo L, Storch E. Strategies for navigating life for parents and siblings of boys with Barth syndrome [Internet]. Larchmonth (NY): Barth Syndrome Foundation. 5 May 2008 [23 November 2017] Available from: https://www.barthsyndrome.org/file_download/ e4a83631-72b0-42f0-894d-0ae70110a6a9.

15. Upton P, Lawford J, Eiser C. Parent-child agreement across child health-related quality of life instruments: A review of the literature. Qual Life Res. 2008; 17(6): 895-913.
16. Kaplan RM. Quality of life measurement. In P. Karoly (Ed.), Measurement Strategies in Health Psychology. New York (NY) John Wiley. 1985.

17. Eiser C, Varni JW. Health-related quality of life and symptom reporting: similarities and differences between children and their parents. Eur J Pediatr. 2013; 172(10): 1299-1304. https://doi.org/10.1007/ s00431-013-2049-9

18. Varni JW, Limbers CA, Burwinkle TM. Parent proxy-report of their children's health-related quality of life: an analysis of 13,878 parents' reliability and validity across age subgroups using the PedsQL ${ }^{\mathrm{TM}} 4.0$ Generic Core Scales. Health Qual Life Outcomes. 207; 5(1): 2.

19. Patel BJ, Lai L, Goldfield G, et al. Psychosocial health and quality of life among children with cardiac diagnoses: agreement and discrepancies between parent and child reports. Cardiol Young. 2017; 27(4): 713721. https://doi.org/10.1017/S1047951116001141

20. Quitmann J, Rohenkohl A, Sommer R, et al. Explaining parentchild (dis)agreement in generic and short stature-specific healthrelated quality of life reports: do family and social relationships matter? Health Qual Life Outcomes. 2016; 14(1): 150.

21. Sattoe JN, van Staa A, Moll HA. The proxy problem anatomized: Child-parent disagreement in health related quality of life reports of chronically ill adolescents. Health Qual Life Outcomes. 2012; 10(1): 10. https://doi.org/10.1186/1477-7525-10-10

22. 22. Dey M, Landolt MA, Mohler-Kuo M. Assessing parent-child agreement in health-related quality of life among three health status groups. Soc Psychiatry Psychiatr Epidemiol. 2013; 48(3): 503-511. https://doi.org/10.1007/s00127-012-0556-z

23. Yi-Frazier JP, Hilliard ME, Fino NF, et al. Whose quality of life is it anyway? Discrepancies between youth and parent health-related quality of life ratings in type 1 and type 2 diabetes. Qual Life Res. 2016; 25(5): 1113-1121. https://doi.org/10.1007/s11136-015$1158-5$

24. Ingerski LM, Modi AC, Hood KK, et al. Health-related quality of life across pediatric chronic conditions. J of Pediatr. 2010; 156(4): 639644. https://doi.org/10.1016/j.jpeds.2009.11.008

25. Vetter TR, Bridgewater CL, McGwin G Jr. An observational study of patient versus parental perceptions of health-related quality of life in children and adolescents with a chronic pain condition: who should the clinician believe?. Health Qual Life Outcomes. 2012; 10(1): 85. https://doi.org/10.1186/1477-7525-10-85

26. Longo E, Badia M, Begoña Orgaz M, et al. Comparing parent and child reports of health-related quality of life and their relationship with leisure participation in children and adolescents with Cerebral Palsy. Res Dev Disabil. 2017; 71: 214-222. https://doi.org/10.1016/j. ridd.2017.09.020

27. White-Koning M, Arnaud C, Dickinson HO, et al. Determinants of child-parent agreement in quality-of-life reports: a European study of children with cerebral palsy. Pediatr. 2007; 120(4): e804-e814.

28. Eiser C, Eiser JR, Stride CB. Quality of life in children newly diagnosed with cancer and their mothers. Health Qual Life Outcomes. 2005; 3(1): 29.

29. Davis E, Mackinnon A, Waters E. Parent proxy-reported quality of life for children with cerebral palsy: is it related to parental psychosocial distress?. Child Care Health Dev. 2012; 38(4): 553-560. https://doi. org/10.1111/j.1365-2214.2011.01267.x

30. Varni JW, Seid M, Rode CA. The PedsQL $L^{\mathrm{TM}}$ : measurement model for the pediatric quality of life inventory. Med Care. 1999; 126-139.

31. Varni JW, Seid M, Kurtin PS. PedsQL $L^{\text {TM }} 4.0$ : Reliability and validity of the Pediatric Quality of Life Inventory ${ }^{\mathrm{TM}}$ Version 4.0 Generic Core Scales in healthy and patient populations. Med Care. 2001; 800-812.

32. Huang IC, Thompson LA, Chi YY, et al. The linkage between pediatric 
quality of life and health conditions: establishing clinically meaningful cutoff scores for the PedsQL. Value Health. 2009; 12(5): 773-781.

33. Varni JW, Sherman SA, Burwinkle TM, et al. The PedsQL $L^{\mathrm{TM}}$ Family Impact Module: Preliminary reliability and validity. Health Qual Life Outcomes. 2004; 2(1): 55.

34. Jastrowski Mano KE, Khan KA, Ladwig RJ, et al. The impact of pediatric chronic pain on parents' health-related quality of life and family functioning: reliability and validity of the PedsQL 4.0 Family Impact Module. J Pediatr Psychol. 2009; 36(5): 517-527.

35. Sheffler LC, Hanley C, Bagley A, et al. Comparison of self-reports and parent proxy-reports of function and quality of life of children with below-the-elbow deficiency. J Bone Joint Surg Am. 2009; 91(12): 2852-2859.

36. Havermans T, Vreys M, Proesmans M, et al. Assessment of agreement between parents and children on health-related quality of life in children with cystic fibrosis. Child Care Health Dev. 2006; 32(1): 1-7.

37. Klassen AF, Miller A, Fine S. Agreement between parent and child report of quality of life in children with attention-deficit/hyperactivity disorder. Child Care Health Dev. 2006; 32(4): 397-406.

38. Youssef NN, Murphy TG, Langseder AL, et al. Quality of life for children with functional abdominal pain: a comparison study of patients' and parents' perceptions. Pediatr. 2006; 117(1): 54-59.

39. Lim Y, Velozo C, Bendixen RM. The level of agreement between child self-reports and parent proxy-reports of health-related quality of life in boys with Duchenne muscular dystrophy. Qual Life Res. 2014; 23(7): 1945-1952.

40. Bray P, Bundy AC, Ryan MM, et al. Health-related quality of life in boys with Duchenne muscular dystrophy: Agreement between parents and their sons. J Child Neurol. 2010; 25(10): 1188-1194.

41. Van der Linden FAH, Kragt JJ, Hobart JC, et al. Proxy measurements in multiple sclerosis: Agreement between patients and their partners on the impact of multiple sclerosis in daily life. J Neurol Neurosurg Psychiatry. 2006; 77(10): 1157-1162.

42. Meijer SA, Sinnema G, Bijstra J0, et al. Social functioning in children with a chronic illness. J Child Psychol Psychiatry. 2000; 41(3): 309317.

43. Levi RB, Drotar D. Health-related quality of life in childhood cancer: Discrepancy in parent-child reports. Int J Cancer. 1999; 83(S12): 5864.

44. Bastiaansen D, Koot HM, Ferdinand RF. Determinants of quality of life in children with psychiatric disorders. Qual Life Res. 2005; 14(6): 1599-1612.

45. Waters E, Doyle J, Wolfe R, et al. Influence of parental gender and selfreported health and illness on parent-reported child health. Pediatr. 2000; 106(6): 1422-1428.

46. Al-Yateem N. Providing Patient Centered Care for Adolescents with Chronic Conditions. Int J Pers Cent Med. 2016; 6(1): 22-29. 